\title{
A Simple Method for Synthesis of Active Esters of Isonicotinic and Picolinic Acids
}

\author{
Jørn B. Christensen* \\ CISMI, University of Copenhagen, Symbion Science Park, Fruebjergvej 3, DK-2100 Copenhagen, \\ Denmark.
}

* Author to whom correspondence should be addressed. E-mail: j.b.Christensen@symbion.ki.ku.dk

Received: 14 May 2000; in revised form 20 December 2000 / Accepted: 20 December 2000 /

Published: 16 January 2001

\begin{abstract}
A method for preparation of the p-nitrophenyl-, N-hydroxysuccinimidyl- and pentafluorophenyl esters of isonicotinic and picolinic acids from the corresponding acids is reported.
\end{abstract}

Keywords: Active esters, pyridine carboxylic acids.

\section{Introduction}

In connection with ongoing synthetic work, we needed access to good acylating reagents based on isonicotinic and picolinic acids. Acylations with isonicotinoyl and picolinoyl chlorides were found to be troublesome, because the acid chlorides are only prepared or available as the corresponding hydrochlorides, which are only sparingly soluble in inert solvents. A further complication was, that upon reaction with polyamines such as poly(propyleneimine) dendrimers incomplete reactions occurred due to extensive salt formation. Therefore we turned our attention to the so called active esters [1].

\section{Results and Discussion}

Searching the literature we found references to the p-nitrophenyl esters of isonicotinic $[2,3]$ and picolinic acid $[2,3,4]$, which have been used for the synthesis of potent antagonists of the luteinizing hormone-releasing hormone (LHRH) [4,5]. The only other ester reported was the pentachlorophenyl 
ester of isonicotinic acid [6], however in the latter case, no experimental details were given. All the procedures were based on the DCC coupling of the acids with the corresponding phenol, however especially in the case of picolinic acid the coupling reaction was hampered by the well known rearrangement of the intermediate $\mathrm{O}$-acylureas to $\mathrm{N}$-acylureas [2]. This problem could be solved by performing the coupling reaction in $\mathrm{CH}_{2} \mathrm{Cl}_{2}$, but again no experimental details were given [2]. We were primarily interested in obtaining the more reactive [1] $\mathrm{N}$-hydroxysuccinimidyl and pentafluorophenyl esters, and since DCC-coupling mainly gave the corresponding $\mathrm{N}$-acyl-N,N'-dicyclohexylureas, the present method was developed. Reaction of the corresponding pyridinecarboxylic acid with $\mathrm{SOCl}_{2}$ catalyzed by DMF [7] at room temperature gives the acid chlorides as their hydrochlorides. These were reacted with 4-nitrophenol, pentafluorphenol and N-hydroxysuccinimide in THF with triethylamine as base to give the corresponding active esters. The compounds prepared are shown Table 1.

Table 1.

Isonicotinic (1) and Picolinic (2) acid esters prepared.

R (yield):

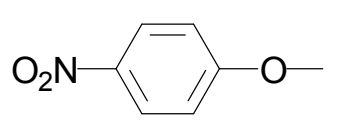<smiles>COc1c(F)c(F)c(F)c(F)c1F</smiles><smiles>CON1C(=O)CCC1=O</smiles><smiles>O=C(O)c1ccncc1</smiles>

1a (54\%)

1b (97\%)

1c $(84 \%)$<smiles>[R]OC(=O)c1ccccn1</smiles>

$2 a(39 \%)$

2b (92\%)

2c $(67 \%)$

\section{Conclusions}

A convenient method has been developed for the synthesis of a variety of active esters of isonicotinic and picolinic acids.

\section{Experimental}

\section{General}

${ }^{1} \mathrm{H}$ - and ${ }^{13} \mathrm{C}$-NMR spectra were obtained using a Varian Gemini 300 NMR or a Varian Unity 400 NMR. Mass spectra were obtained using a Jeol JMS-HX 110 A Tandem Mass Spectrometer. IRspectra were recorded on a Perkin Elmer 1760 X. All melting points are uncorrected. The elemental analyses were performed by Mrs. Karin Linthoe, Department of Organic Chemistry, University of Copenhagen. All chemicals were used as received. 
Isonicotinoylchloride hydrochloride

Thionylchloride $(60 \mathrm{~mL})$ was added carefully to a stirred mixture of isonicotinic acid (24.6 g; 0.2 mol) and DMF (1 mL). A lively gas evolution started, and after 30 minutes all of the acid had gone into solution and the temperature had risen to about $40^{\circ} \mathrm{C}$. Excess thionyl chloride was removed in vacuo, and diethyl ether $(200 \mathrm{~mL})$ was stirred into the residue. The crude product was filtered, washed with diethyl ether and dried in vacuo at $40^{\circ} \mathrm{C}$. Yield: $35.0 \mathrm{~g}$ (98\%) of white crystals sufficiently pure for the preparation of the activated esters.

Picolinoylchloride hydrochloride

Prepared analogously to isonicotinoylchloride hydrochloride.

\section{General procedure for the preparation of active esters}

Isonicotinic acid pentafluorophenyl ester

To a stirred suspension of isonicotinoylchloride hydrochloride $(8.9 \mathrm{~g} ; 0.05 \mathrm{~mol})$ and pentafluorophenol $(9.2 \mathrm{~g} ; 0.05 \mathrm{~mol})$ in THF $(100 \mathrm{~mL})$ was added triethylamine $(20 \mathrm{~mL} ; 0.14 \mathrm{~mol})$ over 10 minutes. The suspension was stirred at room temperature for $12 \mathrm{hrs}$, filtered and concentrated in vacuo. The residue was dissolved in hexane $(300 \mathrm{~mL})$, treated with activated carbon and filtered. The hexane was removed to give the pentafluorophenyl ester as a slightly brownish oil, which crystallized to give a creme colored solid melting $52-54^{\circ} \mathrm{C}$. Yield: $14.0 \mathrm{~g}(97 \%) ;{ }^{1} \mathrm{H}-\mathrm{NMR}\left(300 \mathrm{MHz} ; \mathrm{CDCl}_{3}\right) \delta$ : $8.93(\mathrm{~m} ; 2 \mathrm{H}) ; 8.01(\mathrm{~m} ; 2 \mathrm{H}) .{ }^{13} \mathrm{C}-\mathrm{NMR}\left(100 \mathrm{MHz} ; \mathrm{CDCl}_{3}\right) \delta: 161.2 ; 150.9 ; 142.3(\mathrm{~m}) ; 141.1(\mathrm{~m})$; $139.8(\mathrm{~m}) ; 139.2(\mathrm{~m}) ; 138.5(\mathrm{~m}) ; 136.6(\mathrm{~m}) ; 134.2 ; 123.3 . \mathrm{MS}(\mathrm{EI}): \mathrm{M} / \mathrm{Z}: 290\left(\mathrm{M}^{+}\right) ; 155 ; 106 ; 78 ; 51$. IR (KBr pellet): 3132, 3087, 3063, 2644, 2132, 1765, 1741, 1651, 1592, 1520, 1457, 1436, 1391, 1298, 1241, 1163, 1098, 1067, 1042, 980, 865, 821, 787, 785, 714, 691, 621, 608. An analytical sample was recrystallized from hexane. Found: C: $49.31 ; \mathrm{H}: 1.40$; $: 4.79$. (Calcd. for $\mathrm{C}_{12} \mathrm{H}_{4} \mathrm{~F}_{5} \mathrm{NO}_{2}$ : 49.84\% C; $1.39 \% \mathrm{H} ; 4.84 \%$ N.)

Isonicotinic acid 4-nitrophenyl ester

This compound was prepared according to the general procedure with the exception, that the crude product was crystallized from 2-propanol to give the ester in $54 \%$ yield. $\mathrm{Mp} .137-138^{\circ} \mathrm{C}\left(137-139^{\circ} \mathrm{C}\right.$ [5]). ${ }^{1} \mathrm{H}-\mathrm{NMR}\left(300 \mathrm{MHz} ; \mathrm{CDCl}_{3}\right) \delta: 8.88(\mathrm{~m} ; 2 \mathrm{H}) ; 8.35(\mathrm{~m} ; 2 \mathrm{H}) ; 7.99(\mathrm{~m} ; 2 \mathrm{H}) ; 7.44(\mathrm{~m} ; 2 \mathrm{H}) .{ }^{13} \mathrm{C}-$ NMR (63 MHz; $\left.\mathrm{CDCl}_{3}\right) \delta: 162.80 ; 154.91 ; 150.88 ; 146.0 ; 135.76 ; 125.31 ; 123.05 ; 122.32 . \mathrm{MS}(\mathrm{EI})$ : M/Z: $245\left(\mathrm{M}^{+}\right) ; 106 ; 78 ; 51$. IR (KBr pellet): 3462, 3116, 3086, 3053, 3024, 1746, 1615, 1594, 1567, 1525, 1489, 1408, 1357, 1326, 1312, 1272, 1210, 1163, 1109, 1087, 1061, 1012, 884, 864, 847, 833, $750,699,677,624$. 
Isonicotinic acid N-hydroxysuccinimidyl ester

Prepared according to the general procedure with the exception that the crude product was crystallized from 2-propanol to give the ester in $84 \%$ yield. Mp. $140-141^{\circ} \mathrm{C} .{ }^{1} \mathrm{H}-\mathrm{NMR}(300 \mathrm{MHz}$; $\left.\mathrm{CDCl}_{3}\right) \delta: 8.81(\mathrm{~m} ; 2 \mathrm{H}) ; 7.87(\mathrm{~m} ; 2 \mathrm{H}) ; 2.88(\mathrm{~d} ; \mathrm{J}=8.6 \mathrm{~Hz} ; 4 \mathrm{H}) .{ }^{13} \mathrm{C}-\mathrm{NMR}\left(63 \mathrm{MHz} ; \mathrm{CDCl}_{3}\right) \delta$ : 168.54; 160.62; 150.79; 132.39; 122.95; 25.46. MS(EI): M/Z: $220\left(\mathrm{M}^{+}\right) ; 106 ; 78 ; 51$. Found C: 54.14; H: 3.80; N: 12.49. IR (KBr pellet): 3501, 3064, 2991, 2939, 1806, 1779, 1736, 1596, 1563, 1409, 1367, 1326, 1238, 1201, 1074, 1018, 844, 806, 747, 698, 651, 603. (Calcd. for $\mathrm{C}_{10} \mathrm{H}_{8} \mathrm{~N}_{2} \mathrm{O}_{2}: 54.55 . \%$ C; $3.66 \% \mathrm{H} ; 12.72 \% \mathrm{~N}$.)

Picolinic pentafluorophenyl ester

Prepared according to the general procedure, and crystallized twice from hexane. Transparent crystals. Yield: $92 \%$. Mp. $61-63^{\circ} \mathrm{C} .{ }^{1} \mathrm{H}-\mathrm{NMR}\left(300 \mathrm{MHz} ; \mathrm{CDCl}_{3}\right) \delta: 8.78(\mathrm{~m} ; 1 \mathrm{H}) ; 8.21(\mathrm{~m} ; 1 \mathrm{H}) ; 7.89$ $(\mathrm{m} ; 1 \mathrm{H}) ; 7.56(\mathrm{~m} ; 1 \mathrm{H}) .{ }^{13} \mathrm{C}-\mathrm{NMR}\left(100 \mathrm{MHz} ; \mathrm{CDCl}_{3}\right) \delta: 160.93 ; 150.14 ; 144.89 ; 140.75 ; 139.80$; 139.00; 138.20; 137.29; 136.50; 128.11; 126.38. MS(EI): M/Z: $290\left(\mathrm{M}^{+}\right) ; 242 ; 212 ; 183 ; 155 ; 106 ; 78$. IR (KBr pellet): 3518, 3054, 2467, 1768, 1715, 1654, 1616, 1593, 1520, 1473, 1411, 1330, 1263, 1212, $1161,1078,1056,995,857,845,806,764,748,728,699,622,576$.

Picolinic acid p-nitrophenyl ester

Prepared according to the general procedure, and crystallized from 2-propanol. White needles. Yield: $39 \%$. Mp. $147-149^{\circ} \mathrm{C}$ (dec.). $\left(155-157^{\circ} \mathrm{C}[5]\right) .{ }^{1} \mathrm{H}-\mathrm{NMR}\left(300 \mathrm{MHz} ; \mathrm{CDCl}_{3}\right) \delta: 8.86(\mathrm{~m} ; 1 \mathrm{H})$; $8.31(\mathrm{~m} ; 3 \mathrm{H}) ; 7.96(\mathrm{~m} ; 1 \mathrm{H}) ; 7.60(\mathrm{~m} ; 1 \mathrm{H}) ; 7.45(\mathrm{~m} ; 2 \mathrm{H}) .{ }^{13} \mathrm{C}-\mathrm{NMR}\left(63 \mathrm{MHz} ; \mathrm{CDCl}_{3}\right) \delta: 162.87$; 155.44; 150.13; 146.40; 145.48; 137.25; 127.78; 126.01; 125.16; 122.56; MS (EI): M/Z: $245\left(\mathrm{M}^{+}\right)$; 200; 106; 78. IR (KBr pellet): 3455, 3114, 3079, 1764, 1740, 1615, 1591, 1520, 1489, 1437, 1350, 1293, 1245, 1218, 1160, 1094, 1072, 1045, 995, 887, 865, 836,749, 740, 712, 696, 681, 617.

Picolinic acid N-hydroxysuccinimidyl ester

Prepared according to the general procedure, and crystallized from 2-propanol. White crystals. Yield: $67 \%$. Mp. $172-174{ }^{\circ} \mathrm{C} .{ }^{1} \mathrm{H}-\mathrm{NMR}\left(300 \mathrm{MHz} ; \mathrm{CDCl}_{3}\right) \delta: 8.84(\mathrm{~m} ; 1 \mathrm{H}) ; 8.23(\mathrm{~m} ; 1 \mathrm{H}) ; 8.12(\mathrm{~m} ; 1$ $\mathrm{H}) ; 7.82(\mathrm{~m} ; 1 \mathrm{H}) ; 2.91(\mathrm{~s} ; 4 \mathrm{H}) .{ }^{13} \mathrm{C}-\mathrm{NMR}\left(63 \mathrm{MHz} ; \mathrm{CDCl}_{3}\right) \delta: 169.04 ; 150.06 ; 143.5 ; 137.4 ; 128.5$; 126.2; 25.4. MS (EI): M/Z: $220\left(\mathrm{M}^{+}\right)$; 106; 78. IR (KBr pellet): 3493, 3093, 2955, 1799, 1776, 1742, 1587, 1471, 1440, 1419, 1359, 1265, 1236, 1200, 1066, 1009, 851, 814, 744, 698, 642, 620, 598.

\section{References}

1. Bodanzsky, M. Peptide Chemistry, 2nd ed., Springer-Verlag, Berlin-Heidelberg-New York 1993., pp. 60.

2. Folkers, K.; Ljungqvist, A. The Reaction of Pyridinecarboxylic Acids with Dicyclohexylcarbodiimide and p-Nitrophenol. Acta Chem. Scand. 1988, B42, 408. 
3. Fife, T. H.; Przystas, T. J. Divalent Metal Ion Catalysis in the Hydrolysis of Esters of Picolinic Acid. Metal Ion Promoted Hydroxide Ion and Water Catalyzed Reactions. J. Am. Chem. Soc. 1985, 107, 1041.

4. Flouret, G.; Mahan, K.; Majewski, T. Decreased Histamine Release by Luteinizing HormoneReleasing Hormone Antagonists Obtained upon Translocation of the Cationic Amino Acid from Position 8 to Position 7. J. Med. Chem. 1992, 35, 636.

5. Janecka, A.; Janecki, T.; Shan, S.; Bowers, C.; Folkers, K. Novel, Potent Luteinizing HormoneReleasing Antagonistwith Improved Solubility in Water. J. Med. Chem. 1994, 37, 2238.

6. Solárzano, C.; Davis; M. A. Preparation of Arene Ruthenium(II) Complexes with Activated Ligands for Protein Labeling. Inorg. Chim. Acta 1985, 97, 135.

7. Bosshard, H. H.; Mory, R.; Schmid, M.; Zollinger, H. Eine Methode zur katalysierten Herstellung von Carbonsäure- und Sulfosäure-chloriden mit Thionylchlorid. Helv. Chim. Acta 1959, 42, 1653.

Sample Availability: Available from the author.

(C) 2001 by MDPI (http://www.mdpi.org). Reproduction is permitted for noncommercial purposes. 